\title{
Life-history traits of Drosophila melanogaster populations exhibiting early and late eclosion chronotypes
}

\author{
K. L. Nikhil, Karatgi Ratna and Vijay Kumar Sharma*
}

\begin{abstract}
Background: The hypothesis that circadian clocks confer adaptive advantage to organisms has been proposed based on its ubiquity across almost all levels of complexity and organization of life-forms. This thought has received considerable attention, and studies employing diverse strategies have attempted to investigate it. However, only a handful of them have examined how selection for circadian clock controlled rhythmic behaviors influences lifehistory traits which are known to influence Darwinian fitness. The 'early' and 'late' chronotypes are amongst the most widely studied circadian phenotypes; however, life-history traits associated with these chronotypes, and their consequences on Darwinian fitness remain largely unexplored, primarily due to the lack of a suitable model system. Here we studied several life-history traits of Drosophila melanogaster populations that were subjected to laboratory selection for morning (early) and evening (late) emergence.

Results: We report that the late eclosion chronotypes evolved longer pre-adult duration as compared to the early eclosion chronotypes both under light/dark (LD) and constant dark (DD) conditions, and these differences appear to be mediated by both clock dependent and independent mechanisms. Furthermore, longer pre-adult duration in the late chronotypes does not lead to higher body-mass at pupariation or eclosion, but the late females were significantly more fecund and lived significantly shorter as compared to the early females.

Conclusions: Coevolution of multiple life-history traits in response to selection on timing of eclosion highlights correlations of the genetic architecture governing timing of eclosion with that of fitness components which suggests that timing ecologically relevant behaviors at specific time of the day might confer adaptive advantage.
\end{abstract}

Keywords: Circadian, Adaptive significance, Fitness, Laboratory selection, Life-history evolution

\section{Background}

It is believed that circadian timekeeping mechanisms underlying rhythmic processes provide adaptive advantage to organisms [1-7], and this has prompted studies employing a variety of strategies to examine the adaptive benefits of possessing functional circadian clocks. Surgical ablation of the mammalian 'master circadian clock' suprachiasmatic nucleus [8], and genetic manipulation of circadian clocks in fruit flies Drosophila melanogaster [9] which are known to drive loss of rhythmicity in several key circadian behaviors result in reduced

\footnotetext{
* Correspondence: vsharma@jncasr.ac.in

Chronobiology Laboratory, Evolutionary and Organismal Biology Unit, Jawaharlal Nehru Centre for Advanced Scientific Research, Jakkur, PO Box. 6436, Bangalore, Karnataka 560064, India
}

survivorship [10-13]. Environmentally induced or naturally occurring circadian dysfunction has also been reported to reduce longevity in D. melanogaster $[14,15]$. Beaver et al. [16, 17] reported that D. melanogaster strains carrying loss-of-function mutation in two core clock genes exhibit reduced reproductive output. In addition, studies on organisms inhabiting different latitudes, as well as those living in constant conditions reported large variation in circadian phenotypes in accordance to their local habitats, suggesting that the underlying clocks may have evolved as an adaptation to the presence or absence of cyclic environmental conditions [6, 18-28]. Nevertheless, conclusions drawn from such studies are limited by the lack of adequate information about the ancestry, population size and history of 
the environmental conditions pertaining to the organism's ecology [6].

The eclosion waveform of D. melanogaster comprises a primary peak at dawn (under natural conditions) or around night-day transition (under laboratory light/dark cycles) which gradually reduces through the day with little or no eclosion occurring at night (Additional file 1: Figure S1; [9, 29]). This restriction/gating of eclosion primarily around dawn is hypothesized to be an adaptation to avoid desiccation of pharate adults by high temperature and low humidity prevailing during the rest of the day [3], partly supported by the results of a recent study [30]. Laboratory selection approach has been previously adopted to study how circadian clocks evolve in response to selection for time/phase of eclosion. Selection for 'early' and 'late' emerging strains of Drosophila pseudoobscura and moth Pectinophora gossypiella under LD12:12 (12 h of light and dark cycles each) resulted in the evolution of divergent phase of eclosion $(4 \mathrm{~h}$ in $D$. pseudoobscura and $5 \mathrm{~h}$ in P. gossypiella) [31, 32]. As a correlated response, the early flies in both studies evolved longer circadian clock period while the late flies evolved shorter period. However, these studies suffered from some major shortcomings such as lack of population level replication, details of population ancestry and selection protocols employed (population maintenance methodology, population size and sex ratio) which are known to considerably modify the evolutionary trajectories in response to selection; and thus might have led to misinterpretation of the observed responses to selection (reviewed in [6]). Although these studies suggest that circadian clocks might have evolved to ensure temporal order in behavior and physiology thus enhancing Darwinian fitness (reviewed in [6]), our understanding of how selection for timing of clock controlled behaviors influence life-history traits remains nominal.

To explore the evolutionary trajectory of circadian clocks in response to selection for timing of eclosion, we initiated a long-term study on D. melanogaster populations by imposing selection for eclosion during early morning and late evening hours, which is in contrast to the usual time of eclosion in this species. From a set of 4 ancestral control populations we derived 8 populations 4 replicate early populations using flies that eclose early in the morning and 4 replicate late populations using flies that eclose late in the evening (Additional file 1: Figure S2; see materials and methods for detailed selection protocol). Consequently, the early ${ }_{1-4}$ and the late $_{1-4}$ populations evolved significantly higher morning and evening eclosion respectively relative to the control $_{1-4}$ populations, and exhibited several properties analogous to the well-known 'morning/early' and 'evening/late' chronotypes in humans. Similar to the 'early' and the 'late' human chronotypes [33-35], the early and the late
Drosophila populations evolved shorter and longer clock periods respectively with the control populations exhibiting intermediate period [36], and also exhibited diverged photic phase response curves (PRCs) for both eclosion [36] and activity/rest rhythms [37]. These results indicate that circadian clocks of the two sets of populations 'entrain' differently to LD cycles, or in other words, they are differentially sensitive/interact differentially with LD cycles. This is corroborated by the results of a previous study which reported that the early populations are sensitive to light primarily in the evening while the late populations are sensitive to light primarily in the morning [38]. Collectively, these studies suggest that divergent coevolution of circadian clocks in the early and the late populations might mediate differential interaction/entrainment to regulate time of eclosion.

In the present study, we used the early and the late populations to examine genetic correlations between mechanisms that underlie eclosion at a specific time of the day and that of various pre-adult (egg-to-puparium and egg-to-adult duration, egg-to-puparium and egg-toadult survivorship, and puparial dry-weight) as well as adult life-history traits (dry-weight at eclosion, fecundity, pre- and post-fecundity assay dry-weight, and longevity). As discussed earlier, the early and the late eclosion chronotypes have been shown to be associated with different circadian clock period and differential entrainment to LD cycles, and pre-adult traits such as egg-toadult duration is known to be correlated with circadian clock period. Therefore, to assess the relative contribution of circadian clock period and differential entrainment to LD cycles in driving life-history trait differences between the early and the late populations, we performed some of our experiments under both LD12:12 as well as constant darkness (DD). The rationale being that if differences in life-history traits between the early and the late populations are solely determined by circadian clock period as can be observed under DD when the clock is not under the influence of LD cycles, such differences would either decrease or cease to exist because clock period of all the populations would be held at $24 \mathrm{~h}$ in LD 12:12 by virtue of entrainment [8]. Persistence of differences between populations under both light regimes would imply that the observed life-history trait differences are also driven by clock independent mechanisms.

As mentioned earlier, since $D$. melanogaster eclose predominantly during 'dawn', eclosion at other times of the day is considered to be maladaptive (Additional file 1: Figure S1; [3]). If this is true, then the proportion of individuals which normally eclose early in the morning in the control populations might also differ in terms of fitness from those that eclose late in the evening. To test for such a possibility, one generation before the assays 
we derived 8 additional populations from the controls 4 populations comprising individuals emerging early in the morning, referred to as the early-control, and similarly, 4 populations comprising individuals emerging late in the evening, referred to as the late-control. Also, the early-control and the late-control populations are likely to reveal whether the observed differences in fitness measures between the early and the late populations (if any) are indeed evolved responses to the selection imposed on the timing of eclosion, or are merely environment-driven.

We report that the late populations have evolved significantly longer median egg-to-puparium duration leading to longer egg-to-adult duration, are more fecund around day 11 post-emergence which is the usual day for egg collection as per the selection protocol (see materials and methods), and also exhibit reduced median longevity as compared to the early populations, whereas the early-control and the late-control populations did not differ in the aforesaid life-history traits thus suggesting that the observed differences between the selected populations (early and late) are evolutionary responses to selection for timing of eclosion. Also, even though the early populations differed significantly from the late populations, they were similar to the control populations for most of the traits assayed, the possible reasons for which are discussed later.

\section{Results}

\section{Egg-to-puparium duration}

ANOVA on median egg-to-puparium duration showed statistically significant effect of population, light regime and population $\times$ light regime interaction (Table 1a). Across light regime comparisons revealed that egg-to-puparium

Table 1 Summary of results of ANOVA on (a) median egg-to-puparium duration, (b) arc-sine square root transformed egg-to-puparium survivorship, (c) median egg-to-adult duration, (d) arc-sine square root transformed egg-to-adult survivorship, (e) dry-weight at pupariation, and $(f)$ dry-weight at eclosion values of all populations under LD12:12 and DD light regimes. Summary of results of ANOVA on ( $g$ ) average eggs laid/female, (h) dry-weight at pre- and post fecundity assay stages, (i) log transformed fecundity per unit dry-weight loss, and (j) median longevity of virgin males and females of all populations under LD12:12

\begin{tabular}{|c|c|c|c|c|c|}
\hline Trait & Effect & $d f$ & MS & $F$ & $p$ \\
\hline \multirow[t]{3}{*}{ (a) median egg-topuparium duration } & population & 4 & 42.60 & 48.80 & $<0.0001$ \\
\hline & light regime & 1 & 704.4 & 2261.7 & $<0.0001$ \\
\hline & population $\times$ light regime & 4 & 5.70 & 3.70 & 0.0346 \\
\hline \multirow[t]{3}{*}{ (b) egg-to-puparium survivorship } & population & 4 & 14.70 & 1.47 & 0.2711 \\
\hline & light regime & 1 & 0.03 & 0.004 & 0.9540 \\
\hline & population $\times$ light regime & 4 & 10.6 & 1.05 & 0.4218 \\
\hline \multirow[t]{3}{*}{ (c) median egg-to-adult duration } & population & 4 & 58.00 & 20.00 & $<0.0001$ \\
\hline & light regime & 1 & 2588 & 2189 & $<0.0002$ \\
\hline & population $\times$ light regime & 4 & 8.00 & 4.00 & 0.0207 \\
\hline \multirow[t]{3}{*}{ (d) egg-to-adult survivorship } & population & 4 & 3.20 & 0.38 & 0.8179 \\
\hline & light regime & 1 & 22.7 & 40.52 & 0.0078 \\
\hline & population $\times$ light regime & 4 & 8.30 & 3.78 & 0.0326 \\
\hline \multirow[t]{3}{*}{ (e) dry-weight at pupariation } & population & 4 & 142 & 4.7 & 0.0167 \\
\hline & light regime & 1 & 13231 & 136.3 & 0.0013 \\
\hline & population $\times$ light regime & 4 & 40 & 00.60 & 0.6407 \\
\hline \multirow[t]{3}{*}{ (f) dry-weight at eclosion } & population & 4 & 71 & 2.4 & 0.1107 \\
\hline & light regime & 1 & 2505 & 150.9 & 0.0011 \\
\hline & population $\times$ light regime & 4 & 34 & 2.0 & 0.1612 \\
\hline (g) eggs laid/female & population & 4 & 9.31 & 24.315 & $<0.0001$ \\
\hline \multirow[t]{3}{*}{ (h) pre- and post-fecundity dry-weight } & population & 4 & 53 & 2.00 & 0.1567 \\
\hline & stage & 1 & 27465 & 6835.6 & $<0.0001$ \\
\hline & population $\times$ stage & 4 & 249 & 18.10 & $<0.0001$ \\
\hline (i) fecundity/unit dry-weight loss & population & 4 & 0.002 & 0.43 & $<0.7782$ \\
\hline \multirow[t]{3}{*}{ (j) longevity } & population & 4 & 17.99 & 17.22 & $<0.0001$ \\
\hline & sex & 1 & 83.45 & 70.88 & 0.0035 \\
\hline & population $\times$ sex & 4 & 11.44 & 5.27 & 0.0011 \\
\hline
\end{tabular}


duration for all the populations was significantly longer (by $8.4 \mathrm{~h}$ or $7 \%$ ) in LD12:12 as compared to that in DD thus highlighting the delaying effect of LD cycles on egg-topuparium duration (Fig. 1a-c; Additional file 1: Figure S3; Additional file 1: Table S1).

In LD12:12, the late populations had a significantly longer (by $6.5 \mathrm{~h}$ or $5.4 \%$ ) egg-to-puparium duration $(129.11 \mathrm{~h})$ as compared to all other populations (early = $122.43 \mathrm{~h}$, early-control $=123.01 \mathrm{~h}$, control $=121.84 \mathrm{~h}$ and late-control $=122.89 \mathrm{~h}$ ) while that for the remaining four sets of populations did not differ among each other (Fig. 1a, c; Additional file 1: Figure S3; Additional file 1: Table S1).

In $\mathrm{DD}$, the late populations took significantly longer (by $5 \mathrm{~h}$ or $3.6 \%$ ) to pupariate $(118.14 \mathrm{~h})$ as compared to the early $(113.57 \mathrm{~h})$ and the control (114.47 $\mathrm{h}$ ) populations but did not differ from the early-control $(114.83 \mathrm{~h})$ and the late-control $(116.32 \mathrm{~h})$ populations, whereas none of the other sets of populations differed among each other (Fig. 1b, c; Additional file 1: Figure S3; Additional file 1: Table S1).

\section{Egg-to-puparium survivorship}

ANOVA on egg-to-puparium survivorship revealed that the effect of population, light regime and population $\times$ light regime interaction was statistically not significant (Table 1b), indicating that the populations did not differ in their egg-to-puparium survivorship both within and across light regimes.

The average egg-to-puparium survivorship across populations was $85.56 \pm 1.24 \%($ mean $\pm \mathrm{SD})$ in LD12:12 (early $=$ $86.86 \%$, early-control $=84.33 \%$, control $=84.60 \%$, latecontrol $=85.08 \%$ and late $=86.93 \%)$ and $85.35 \pm 1.72 \%$ $($ mean $\pm \mathrm{SD})$ in DD (early $=83.56 \%$, early-control $=$ $83.18 \%$, control $=88.03 \%$, late-control $=83.36 \%$ and late $=88.61 \%$; Fig. 1d; Additional file 1: Table S2).

\section{Egg-to-adult duration}

ANOVA on median egg-to-adult duration revealed statistically significant effect of population, light regime and population $\times$ light regime interaction (Table $1 \mathrm{c}$ ). As observed for egg-to-puparium duration, the egg-to-adult duration in LD12:12 was also significantly longer (by $16 \mathrm{~h}$ or $7.5 \%)$ for all the populations as compared to that in DD (Fig. 2a-c; Additional file 1: Figure S4).

In LD12:12, egg-to-adult duration of the late population $(239.15 \mathrm{~h}$ ) was significantly longer (by $4 \mathrm{~h}$ or $1.9 \%$ ) than all populations (early $=234.69 \mathrm{~h}$, early-control $=$ $235.70 \mathrm{~h}$ and late-control $=234.68 \mathrm{~h}$ ) except the control $(236.44 \mathrm{~h})$ populations whereas that of all other populations (early, early-control, control and late-control) did not differ significantly among each other (Fig. 2a, c; Additional file 1: Figure S4; Additional file 1: Table S1). (a)
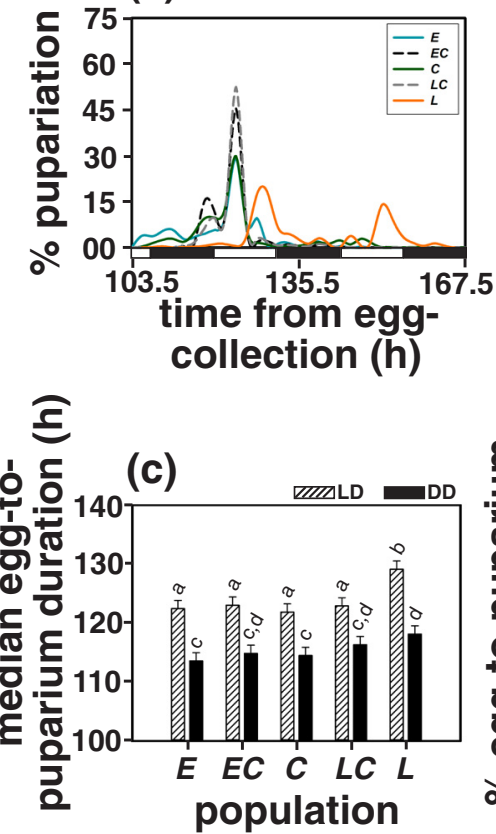

(b)
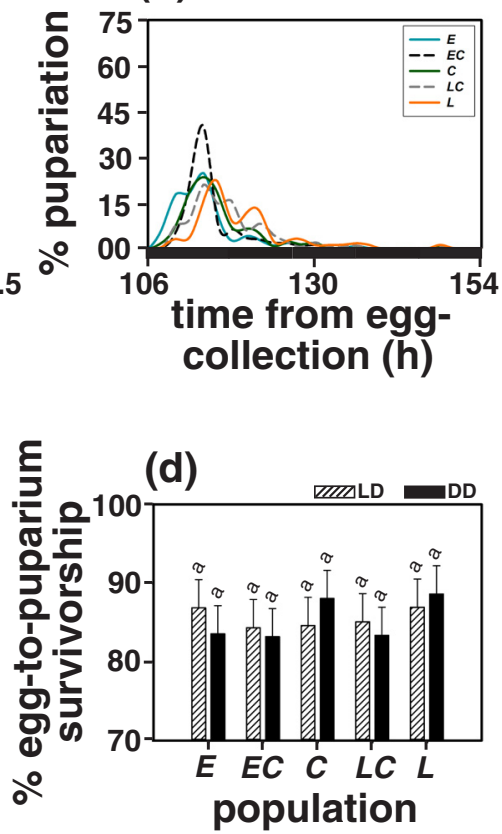

Fig. 1 Percentage pupariation as a function of time (in hours) from egg-collection in a LD12:12 and $\mathbf{b}$ DD for the early (E), the early-control (EC), the control $(C)$, the late-control ( $L C$ and the late $(L)$ populations. The black and white bars at the bottom represent night and day respectively. $\mathbf{c}$ Median egg-to-puparium duration (time taken for $50 \%$ of total pupariation events) of all populations in LD12:12 and DD, and $\mathbf{d}$ Percentage egg-to-puparium survivorship values in LD12:12 and DD. Error bars for panels c and d indicate $95 \%$ confidence intervals calculated by method of Tukey's HSD. Bars sharing same letters do not differ statistically while those with different letters are significantly different from each other 
(a)

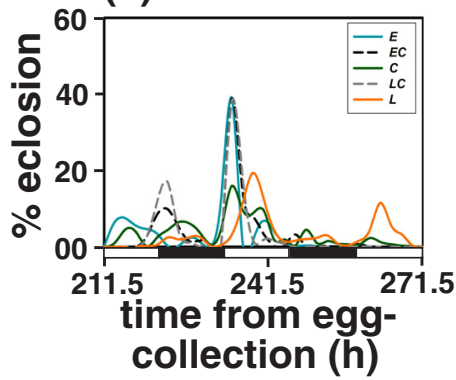

(b)

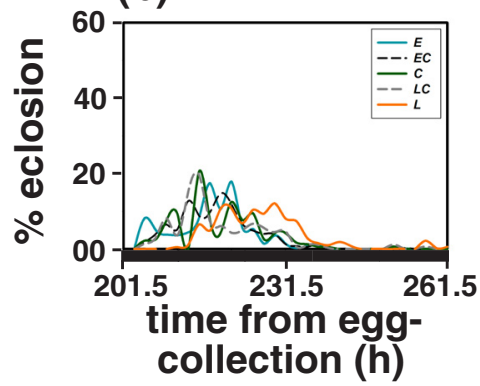

(d)

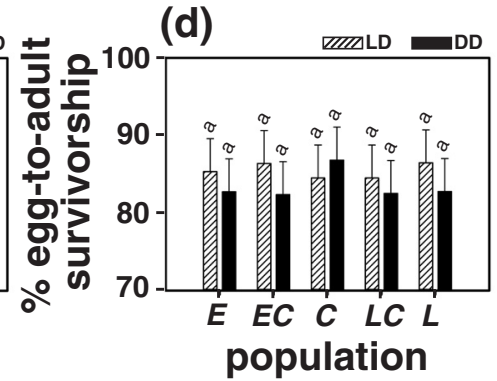

Fig. 2 Percentage of flies eclosing as a function of time (in hours) from egg-collection in a LD12:12 and $\mathbf{b}$ DD for the early (E), the early-control $(E C)$, the control $(C)$, the late-control $(L C)$ and the late $(L)$ populations. The black and white bars at the bottom represent night and day respectively. c Median egg-to-adult duration of all populations in LD12:12 and DD, and $\mathbf{d}$ Percentage egg-to-adult survivorship values of all populations in LD12:12 and DD. All other details are same as in Fig. 1

Under DD, the late populations exhibited a significantly longer (by $8 \mathrm{~h}$ or $3.5 \%$ ) egg-to-adult duration $(226.41 \mathrm{~h})$ as compared to all other populations (early $=218.90 \mathrm{~h}$, early-control $=217.96 \mathrm{~h}$ and latecontrol $=217.79 \mathrm{~h})$ except the controls $(219.18 \mathrm{~h}$; Fig. 2b, c; Additional file 1: Figure S4; Additional file 1: Table S1).

\section{Egg-to-adult survivorship}

ANOVA on egg-to-adult survivorship revealed statistically significant effect of light regime and population $\times$ light regime interaction but not of population (Table 1d). However, post hoc multiple comparisons using Tukey's HSD did not reveal any statistically significant difference in eggto-adult survivorship either across LD12:12 (early= $85.33 \%$, early-control $=86.38 \%$, control $=84.51 \%$, latecontrol $=84.50 \%$ and late $=86.48 \%$ ) and DD (early $=$ $82.74 \%$, early-control $=82.37 \%$, control $=86.82 \%$, latecontrol $=82.52 \%$ and late $=82.76 \%$ ) light regimes or across populations within a light regime (Fig. 2d; Additional file 1: Table S2).

\section{Dry-weight}

Since the late populations exhibited significantly longer egg-to-puparium and egg-to-adult duration, we further tested if this lengthening of pre-adult developmental duration translated to higher dry-weight at pupariation and eclosion.
ANOVA on pupal dry-weight revealed statistically significant effect of population and light regime but not of population $\times$ light regime interaction (Table 1e). In accordance with the difference in egg-to-puparium duration between light regimes, the pupal dry-weight was found to be significantly higher (on an average by $6.3 \%$ ) in LD12:12 (early $=576.16 \mu \mathrm{g}$, early-control $=570.53 \mu \mathrm{g}$, control $=572.17 \mu \mathrm{g}$, late-control $=575.11 \mu \mathrm{g}$ and late $=$ $580.16 \mu$ g; Fig. 3a; Additional file 1: Table S3) as compared to that in DD (early $=533.52 \mu \mathrm{g}$, early-control $=$ $536.46 \mu \mathrm{g}$, control $=533.33 \mu \mathrm{g}$, late-control $=544.07 \mu \mathrm{g}$ and late $=544.83 \mu \mathrm{g}$; Fig. 3a; Additional file 1: Table S3) whereas no difference was observed between populations within either of the light regimes.

ANOVA on dry-weight at eclosion reported statistically significant effect of light regime but not of population or population $\times$ light regime interaction (Table 1f). In accordance with egg-to-adult duration differences across light regimes, dry-weight at eclosion was found to be significantly higher (on an average by $4.35 \%$ ) in LD12:12 (early $=359.39 \mu \mathrm{g}$, early-control $=358.30 \mu \mathrm{g}$, control $=361.71 \mu \mathrm{g}$, late-control $=362.64 \mu \mathrm{g}$ and late $=$ $369.94 \mu \mathrm{g}$; Fig. 3b; Additional file 1: Table S3) as compared to that in DD (early $=342.63 \mu \mathrm{g}$, early-control $=$ $347.85 \mu \mathrm{g}$, control $=346.19 \mu \mathrm{g}$, late-control $=348.12 \mu \mathrm{g}$ and late $=348.06 \mu \mathrm{g}$; Fig. 3b; Additional file 1: Table S3) whereas the populations did not differ among each other in either of the light regimes. 
(a) puparium

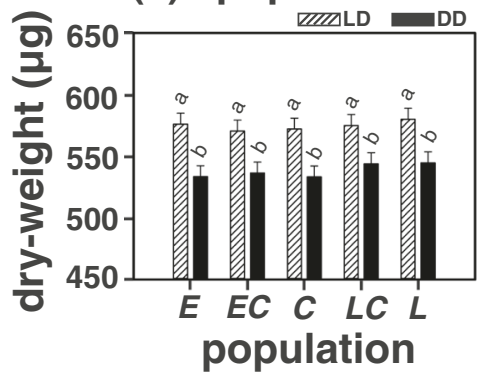

(b) adult

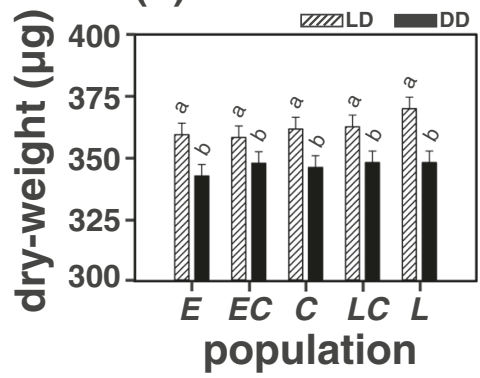

Fig. 3 Dry-weight per individual at a pupariation, and $\mathbf{b}$ eclosion for the early (E), the early-Control (EC), the control (C), the late-control ( $L C)$ and the late (L) populations in LD12:12 and DD light regimes. All other details are same as in Fig. 1

\section{Fecundity}

ANOVA on average fecundity data revealed a statistically significant effect of population (Table 1g). Fecundity of the late populations (10.80 eggs/fly) was significantly higher (by $32 \%$ ) as compared to that of the other populations (early= $7.32 \mathrm{eggs} / \mathrm{fly}$, early-control $=7.74 \mathrm{eggs} / \mathrm{fly}$, control $=7.01$ eggs/fly and late-control $=7.68$ eggs/fly), whereas none of the other populations differed significantly among each other (Fig. 4a; Additional file 1: Table S4).

\section{Pre- and post-fecundity assay dry-weights}

ANOVA on female dry-weight measurements at pre- and post-fecundity assay stages showed statistically significant effect of stage (pre/post-fecundity assay) and population $x$ stage interaction but not of population (Table 1h). Post hoc multiple comparisons revealed that post-fecundity assay dry-weight of all the populations was reduced by about $52.40 \mu \mathrm{g}$ (22 \%; Fig. 4b) as compared to the prefecundity assay dry-weight. Pre-fecundity assay dry-weight did not differ statistically between populations (early= $231.95 \mu \mathrm{g}$, early-control $=233.62 \mu \mathrm{g}$, control $=229.95 \mu \mathrm{g}$, late-control $=232.87 \mu \mathrm{g}$ and late $=238.45 \mu \mathrm{g}$; Table $1 \mathrm{~h}$; Additional file 1: Table S4) but post-fecundity assay dryweight of the late populations $(167.16 \mu \mathrm{g})$ was significantly lower (by $17 \mu \mathrm{g}$ or $\sim 10 \%$ ) as compared to that of all other populations $($ early $=182.79 \mu \mathrm{g}$, early-control $=184.30 \mu \mathrm{g}$,

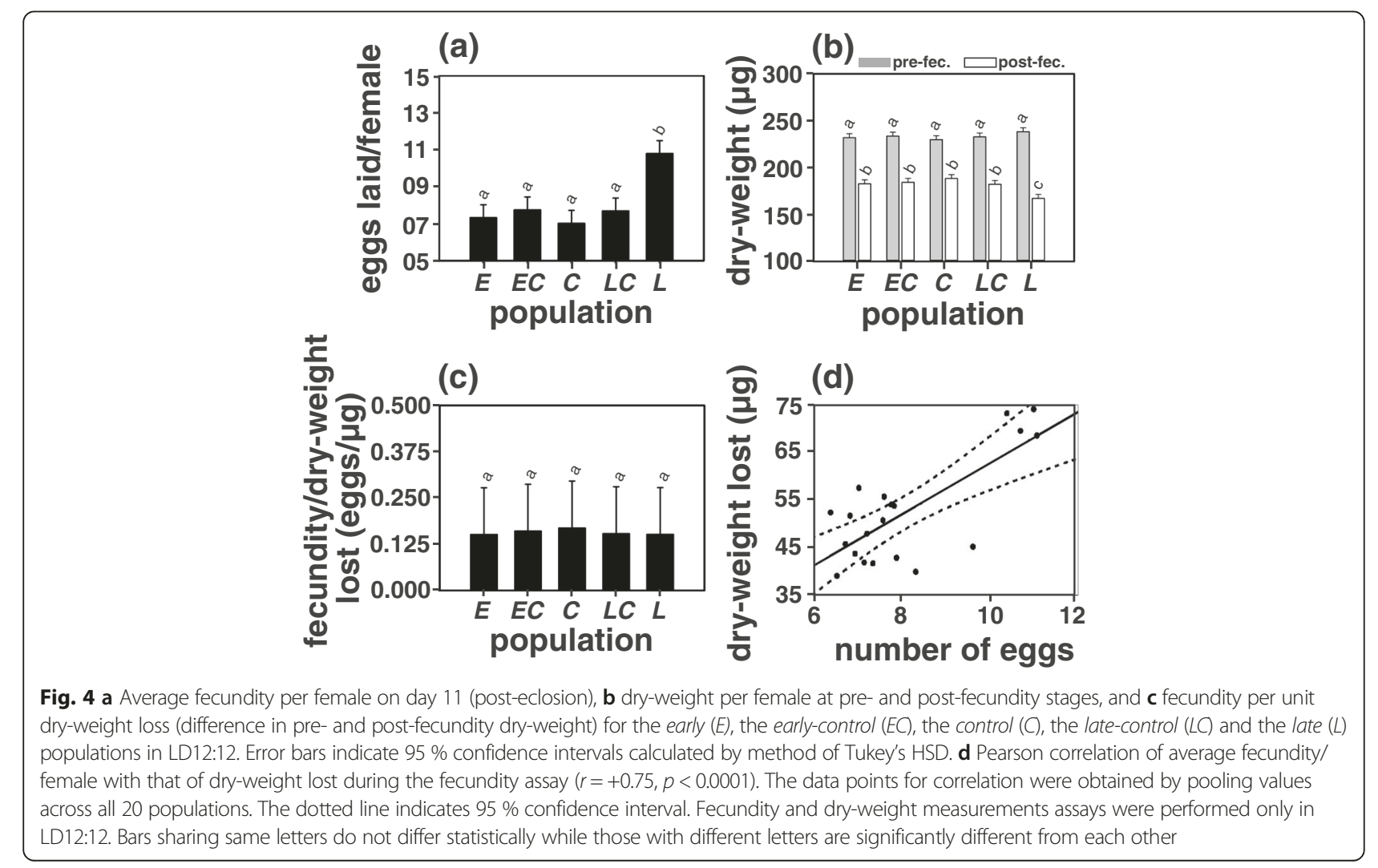


control $=188.41 \mu \mathrm{g}$ and late-control $=182.16 \mu \mathrm{g}$; Fig. 4b; Table 1h; Additional file 1: Table S4).

\section{Fecundity per unit loss in dry-weight}

When normalized by the dry-weight lost (difference in pre- and post-fecundity assay dry-weight), fecundity per unit dry-weight lost did not differ statistically across populations (early $=0.15$ eggs $/ \mu \mathrm{g}$, early-control $=0.16$ eggs $/ \mu \mathrm{g}$, control $=0.17 \mathrm{eggs} / \mu \mathrm{g}$, late-control $=0.15 \mathrm{eggs} /$ $\mu \mathrm{g}$ and late $=0.15$ eggs/ $\mu \mathrm{g}$; Fig. $4 \mathrm{c}$; Table 1i), suggesting that although the late populations were more fecund they lose more dry-weight due to the higher number of eggs laid. As an additional confirmation of this, we performed a linear correlation between egg output and dryweight loss by pooling data from all the populations, and found that the two were significantly positively correlated $(r=+0.75, p<0.0001$; Fig. $4 \mathrm{~d})$.

\section{Longevity}

ANOVA on median longevity reported statistically significant effect of population, sex and population $\times$ sex interaction (Table 1j). With the exception of the late populations where individuals of both the sexes had an average median longevity of $41.89 \pm 0.004$ days (mean \pm $\mathrm{SD}$ ), the average female longevity of all the other populations (early $=47.5$ days, early-control $=46.38$ days, control $=47.81$ days and late-control $=45.22$ days; Fig. 5; Additional file 1: Table S4) was $\sim 7 \%$ higher than that of the males (early $=41.41$ days, early-control $=42.67$ days, control $=44.25$ days and late-control $=44.13$ days; Fig. 5; Additional file 1: Table S4).

Within sex comparisons revealed that females of the late populations exhibited significantly shorter $(\sim 12 \%)$ median longevity as compared to females of the other populations with the exception of the late-control females which did not differ statistically from the late populations. The median male longevity was not observed to differ statistically across populations (Fig. 5; Additional file 1: Table S4).

\section{Discussion}

We observe that the late populations evolved longer egg-to-puparium and egg-to-adult duration as compared to the early populations thus highlighting an association between eclosion chronotype and pre-adult developmental duration (Figs. 1a-c, 2a-c). Under LD12:12, the difference in median egg-to-puparium duration between the late and the early populations is $\sim 7 \mathrm{~h}$, which is reduced to $\sim 3 \mathrm{~h}$ at eclosion (Figs. 1c, 2c). One possible reason for this might be a genotype dependent effect of light on pupal development as also suggested by the median eggto-puarium duration of the late populations which differs from all other populations under LD12:12 but not DD (Fig. 1c). Alternatively, under LD cycles, timing of eclosion is known to be governed by a circadian clock component as well as a clock independent masking response to lights-ON as discussed with respect to the same populations in a previous study [39]. Thus, in addition to the clock determined time of eclosion,
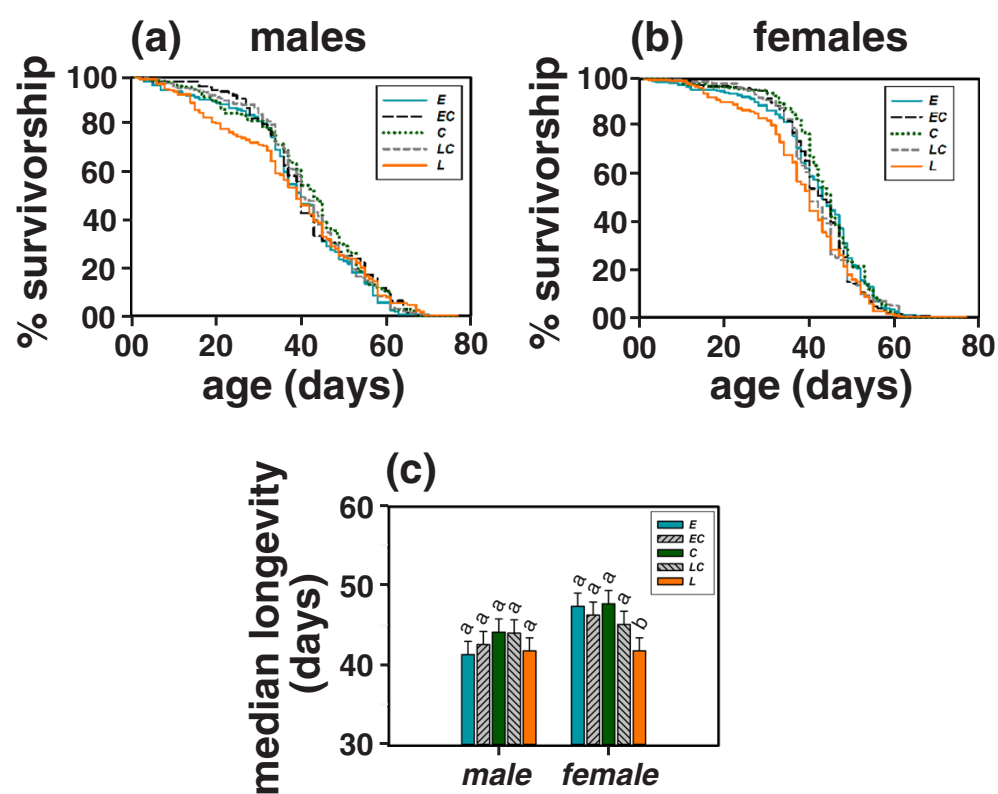

Fig. 5 Survivorship curves of a virgin males and $\mathbf{b}$ virgin females of the early $(E)$, the early-control (EC), the control (C), the late-control (LC) and the late (L) populations in LD12:12. c Median longevity (time taken for $50 \%$ of individuals to die) of virgin males and females of all populations in LD12:12. All other details are same as in Fig. 1 
masking response to light, which results in an additional burst of eclosion immediately following lights-ON might have reduced the median pre-adult duration. This is further supported by our observation under DD that $\sim 4 \mathrm{~h}$ difference in egg-to-puparium duration between the late and the early populations increases to $\sim 7 \mathrm{~h}$ at eclosion (Figs. 1c, 2c). Thus, the observed reduction in median egg-to-adult duration under LD12:12 may be a result of the combination of both (a) artefact of masking response to lights-ON which is clearly absent under DD and (b) differential effect of light on pupal development, which remains to be addressed further. In addition to the divergent eclosion chronotypes, the early and the late populations have also evolved shorter and longer clock periods differing by $40 \mathrm{~min}[36,39]$ which suggests a correlation between emergence chronotype and circadian clock period. Such correlations have been reported earlier in the melon fly, Bactrocera cucurbitae [40, 41], and between clock period and egg-to-adult duration in fruit flies D. melanogaster [42-44], suggesting that clock period differences influence pre-adult developmental rates. In DD, egg-to-puparium duration of the late populations was $118.14 \mathrm{~h}$ (4.9 days) and egg-to-adult duration was $226.41 \mathrm{~h}$ (9.4 days) as opposed to $113.56 \mathrm{~h}$ (4.7 days) and $218.90 \mathrm{~h}$ (9.1 days) respectively of the early populations. If the pre-adult duration of the early and the late populations was entirely driven by circadian clock period difference, under DD the early and the late populations would drift apart by $0.66 \mathrm{~h}$ (40 min) every day, and consequently the two populations would exhibit a $3.12 \mathrm{~h}$ difference in egg-to-puparium duration (in 4.7 days which is equal to the time taken by the early population to pupariate) and $6.01 \mathrm{~h}$ difference in egg-to-adult duration (in 9.12 days) which is considerably smaller than that observed empirically (Figs. 1a, c; 2a, c). Since eggs for the egg-to-puparium and egg-to-adult duration assays were collected from all populations at the same time (thus were age matched), the observed differences in pre-adult duration between the early and the late populations are unlikely to be due to the differences in the age of eggs. Moreover, the time of egg-collection or the age of eggs does not alter the difference in egg-toadult duration between the early and the late populations [45]. Taken together these results suggest that difference in pre-adult developmental rates of the early and the late populations is not entirely circadian clock driven, and may also involve clock independent mechanisms which might drive differential interaction with LD cycles (significant population $\times$ light regime interaction reported in Table 1a, c).

Furthermore, light mediated enhancement in the preadult developmental rates is apparent as both egg-topuparium and egg-to-adult duration of all the populations was 7-7.5 \% longer in LD12:12 as compared to DD
(Figs. 1a-c, 2a-c; Additional file 1: Figure S3, Additional file 1: Figure S4; Additional file 1: Table S1). While effect of light on pre-adult duration has been documented earlier $[46,47]$, precise mechanisms underlying such effects remains to be explored. The timing of eclosion in Drosophila is believed to depend upon a number of factors including the developmental state of the fly, the phase and period of circadian rhythm, hormonal cascade, and environmental condition $[48,49]$. The LD cycles interact with the circadian clock controlled gate of eclosion such that even if flies have completed development, they are allowed to eclose only during certain time of the day and not merely in accordance with their developmental state, and consequently the time of eclosion is delayed in LD12:12 as compared to DD [47-50]. Additionally, the time of eclosion on a given day is also a function of the circadian clock period such that individuals with shorter period eclose earlier than those with longer clock period [51]. This further supports the notion that pre-adult development in D. melanogaster is probably mediated by the interaction of circadian clocks with LD cycles.

Differences in pre-adult developmental rates of the early and the late populations do not seem to influence their egg-to-puparium and egg-to-adult survivorship; nor do the light regime mediated differences affect pre-adult survivorship (Figs. 1d, 2d; Additional file 1: Table S2). This might be due to the magnitude of difference in egg-topuparium or egg-to-adult duration between the populations not being large enough to influence egg-topuparium and egg-to-adult survivorship.

Although the late populations have evolved longer preadult developmental duration, their body-weight at pupariation and at eclosion did not differ from that of all the other populations (Fig. 3; Additional file 1: Table S3). However, dry-weight of puparia and adults were found to be significantly higher for all the populations in LD12:12 as compared to DD (Fig. 3b; Additional file 1: Table S3) which is not surprising as egg-to-puparium and egg-toadult duration is significantly enhanced in LD12:12 as compared to DD (Figs. 1, 2).

Coevolution of pre-adult life-history traits in response to selection for timing of eclosion is intuitive, as changes in pre-adult stages can directly affect the time course and waveform of eclosion. It would be interesting to know whether selection for eclosion at different time of the day also led to correlated changes in adult life-history traits that may not necessarily influence eclosion time but would highlight the underlying genetic correlation. In this regard, we observed that the late populations exhibited a significantly higher fecundity as compared to all other populations (Fig. 4a; Additional file 1: Table S4). In D. melanogaster, pre-adult developmental duration is known to be correlated with fecundity, and delayed development is associated with higher dry-weight, and in turn with higher 
fecundity [52-54]. This does not appear to be the case in the late populations since neither their dry-weight at eclosion nor pre-fecundity assay dry-weight differed from the other populations (Fig. 4b; Additional file 1: Table S4). However, post-fecundity assay dry-weight of the late populations was significantly lower as compared to that of the other populations (Fig. 4b; Additional file 1: Table S4), but when normalized by the loss of dry-weight (difference in pre- and post-fecundity assay dry-weight), fecundity per unit dry-weight lost was similar for all populations (Fig. 4c). Therefore, a significant reduction in postfecundity assay dry-weight in the late populations appears to be a consequence of higher number of eggs laid as also substantiated by a significant correlation observed between the number of eggs laid and dry-weight lost (Fig. 4d). Therefore, contrary to the well-known positive correlation between pre-adult developmental duration, dry-weight and fecundity, our results suggest that the observed higher fecundity in the late populations is not due to higher dry-weight attained because of the delay in the timing of eclosion but may be due to other mechanisms such as pleiotropy or mutation accumulation [55]. Alternatively, higher fecundity in the late populations might have evolved as an artefact of the nature of selection protocol employed. For instance, to ensure that the number of adults in all populations is $\sim 1200$, every generation we collect larger number of eggs for the late populations followed by relatively smaller number of eggs for the early populations as compared to the control populations (see materials and methods) which is 24 vials per replicate population for the early populations, 48 vials for the late populations as opposed to 16 vials for the control populations with each vial housing approximately 300 eggs. Therefore, the number of eggs collected from the late populations ( 14400 eggs) is approximately twice that of the early ( 7200 eggs) and thrice that of the control ( 4800 eggs) populations. This might have led to an inadvertent selection for higher fecundity in the late populations, and also, possibly as a consequence of higher effective population size $\left(N_{\mathrm{e}}\right)$ the late populations might experience relatively lower extent of inbreeding depression followed by the early populations, with the control populations experiencing highest degree of inbreeding depression. If this were to be true, then the early populations would also be expected to exhibit higher fecundity as compared to the control populations, but that does not seem to be the case. Therefore, it is unlikely that this reasoning can account for the evolution of higher fecundity in the late populations even though it cannot be entirely disregarded. However, the possibility of such a scenario can also be clarified by a cross experiment between the early and the late populations. Additionally, given that fecundity in Drosophila is not constant across lifespan, the difference in fecundity between the populations observed on days 10-12 post-eclosion might also vary across different ages. For instance, in light of the results from a previous study [55], it is also possible that the early populations which exhibit significantly lower fecundity on days 10-12 might otherwise exhibit higher fecundity at an earlier stage and vice versa for the late populations. However, since the fly populations used in the current study are maintained on a non-overlapping 21 day generation cycle (see materials and methods) wherein eggs laid only on the day 11 of adulthood are used for the next generation, only eggs laid around this day determines the populations' fitness in this regime. Therefore, fecundity during other lifestages is irrelevant under the currently discussed regimen but nevertheless will be interesting to examine.

Further, we found that females of the late populations live significantly shorter as compared to those from the early and the control populations, while no difference in longevity was observed between males (Fig. 5a-c). The reduction in longevity of the late females as compared to the early and the control females was consistently observed in four replicate populations maintained under similar environmental conditions. In light of fecundity and dry-weight results, the observed reduction in longevity of the late females appears to represent the classic trade-off between fecundity and adult lifespan due to the antagonistic pleiotropic effects of the underlying genes [56-58]. However, since the results presented here are on virgins, the observed reduction in longevity cannot be explained entirely by such a trade-off. Therefore, even though higher reproductive output may have evolved as an artefact of the selection protocol, reduced longevity in the late females as compared to the early and the control females may have evolved as a correlated response to selection for late evening eclosion and not directly as a consequence of higher fecundity. Interestingly, we also observe that the reduced longevity in the females of the late populations is primarily a consequence of early death (around days 20-40) and not during the later lifestages, and a similar trend is also observed in the males. However, the possible reason for such observations remains to be explored.

Thus, we report that selection for late evening eclosion in fruit flies $D$. melanogaster is associated with the coevolution of several life-history traits in the late populations, while no difference was observed between the early, the early-control and the late-control populations. Such correlations between chronotypes/circadian clocks and life-history traits have been reported earlier. Notably, Yadav and Sharma $[59,60]$ demonstrated that selection for faster pre-adult development leads to the coevolution of shorter clock period, and that the faster developing populations evolve reduced dry-weight, body size, fecundity, starvation and desiccation resistance, and longevity. Similarly, in a separate study on the melon fly 
Bactrocera cucurbitae, selection for egg-to-adult duration resulted in the coevolution of divergent phase of activity/rest and mating rhythms [40]. Most differences observed in our study, however, correspond to the late populations relative to the control populations, while very little difference was observed between the early and the control populations. Also, most of the life-history traits assayed in the late populations differed by a small magnitude varying from $2-10 \%$ as compared to the early and the control populations. This is not surprising considering the larger time difference between the selection window of the late populations from the eclosion peak of the control populations, and proximity of the selection window of the early populations from the eclosion peak of the control populations (see materials and methods). Since evening eclosion is not predominantly seen in the control populations, the late populations would experience a much stronger selection pressure as compared to the early populations which in turn might drive faster coevolution of life-history traits.

In summary, selection for late evening eclosion leads to lengthening of pre-adult duration without any increase in body-weight at eclosion, increased fecundity associated with greater post-fecundity assay dry-weight loss and reduced virgin female longevity. The observed life-history traits in the late populations being evolved responses to selection is further supported by our observation on the early-control and the late-control populations. That life-history traits of the early-control and the late-control populations did not differ significantly from each other but were different from that of the early and the late populations (in most cases) suggests that the observed life-history trait differences between the early and the late populations are evolutionary response to the imposed selection and are not merely environmentally driven. Furthermore, although the pre-adult and adult life-history traits studied here are known to be highly correlated, enhanced fecundity in the late populations does not seem to be a consequence of higher biomass attained by lengthening of egg-to-adult duration. Thus the differences in adult traits do not seem to be associated with pre-adult trait differences and appear to be driven by independent mechanisms that might have evolved as a consequence of selection.

\section{Conclusions}

Thus, in contrast to studies which demonstrated the effect of direct manipulation of circadian clock on fitness aspects (see introduction), we report coevolution of life-history traits in independently evolved replicate populations of $D$. melanogaster exhibiting early and late eclosion chronotypes, suggesting that the genetic architecture underlying eclosion at specific times of the day (eclosion chronotypes) is genetically correlated with several life-history traits, and these correlations appear to encompass both circadian clock-dependent and clock-independent mechanisms. Thus the extent of circadian clocks' influence in the observed trait differences, and the underlying genetic architecture remains to be explored.

\section{Methods \\ Experimental populations and assay conditions}

Additional file 1: Figure S2 presents a schematic of the selection protocol employed to generate the early and the late populations from the control populations. Populations selected for early morning and late evening eclosion comprised four replicates each of the early $y_{\mathrm{i}}$ and the late $_{\mathrm{j}}(i=j=1-4)$ initiated from four replicates of the control $l_{\mathrm{k}}(k=1-4)$ whose ancestry details are provided elsewhere [36]. Briefly, the early and the late populations with a given subscript were derived from the control population with the same subscript, and therefore share a common ancestry. For example, the early $y_{1}$ and the late $_{1}$ populations were initiated from the control $_{1}$ population, and similarly for the other three replicates. Since our study aims at exploring evolutionary trajectories of traits in a population, the unit of biological replication is a population and thus, the four populations of each selection type are biological replicates in all our experiments. All 12 populations (four replicates each for early, control and late) were maintained on a 21 day discrete generation cycle, and flies were housed in plexi-glass cages of dimension $25 \times 20 \times 15 \mathrm{~cm}^{3}$ with $\sim 1200$ individuals per cage (sex ratio $\sim 1: 1$ ), and were provided with $a d$ libitum banana-jaggery (BJ) medium. The parental populations were provided with food supplemented with yeast paste (to boost their fecundity) for three days prior to egg collection, and 2300 eggs were collected and dispensed into each culture vial (16 vials for control, 24 for early and 48 for late populations) containing $\sim 6 \mathrm{ml} \mathrm{BJ}$ medium. From the initiation of eclosion, which is generally on day 9 (at $25{ }^{\circ} \mathrm{C}$ ) post egg collection, flies that eclosed early in the morning between Zeitgeber Time (ZT) 21-01 (ZT00 and ZT12 represents time of lights$\mathrm{ON}$ and lights-OFF respectively under LD12:12) for 3-4 consecutive days were collected to form the early populations, while flies that eclosed late in the evening between ZT09-13 formed the late populations. For the control populations, flies were collected once every $24 \mathrm{~h}$ for the same 3-4 days and thus, comprised individuals emerging throughout these 3-4 days without any selection imposed on timing of eclosion. The days of initiation and termination of fly collection within the respective selection windows was kept constant for all populations. In other words, if collection of flies for the early populations was started on day $\mathrm{x}$ and terminated on day $\mathrm{y}$, collection of flies for the control and the late populations was also initiated and terminated on days ' $\mathrm{x}$ ' 
and ' $y$ ' respectively so as to ensure that the populations are selected only for eclosion at different gate/time of the day and to avoid any unintended selection for faster and slower pre-adult development. The implementation of selection protocol and regular maintenance of populations was performed under LD12:12 with $\sim 0.4 \mathrm{Wm}^{-2}$ light intensity during the light phase, $25 \pm 0.5{ }^{\circ} \mathrm{C}$ temperature, and $75 \pm 5 \%$ relative humidity.

In addition to the four replicate populations each for the early, the control and the late, we used four replicates each for the two other populations (early-control and late-control; see Introduction). From the control populations, flies emerging in the morning window (ZT21-01) were collected to form the early-control populations and similarly, flies emerging in the evening window (ZT09-13) formed the late-control populations. This procedure was implemented on all four replicates of the control populations for only one generation prior to the assays, and therefore, unlike the early and the late populations, the early-control and the late-control populations were not subjected to any long-term selection protocol.

To minimize the effects of non-genetic inheritance (reviewed in [61]) due to different selection regimes, all populations were subjected to one generation of standardization with the maintenance protocol which is identical to that used for the control populations. This was achieved by relaxing selection on timing of eclosion by collecting all flies that eclosed throughout the first 4 days similar to that for the control populations while the population size was maintained at 1200 flies per replicate population. Since the primary purpose of using the early-control and the late-control populations was to asses if the observed differences between the early and the late populations are evolved responses to selection as not merely environmental in origin, these populations were also subjected to standardization by deriving them from the control populations followed by relaxation of selection for one generation as described above. All assays described in the present study were performed on the progeny of the standardized populations at the $242^{\text {nd }}$ generation ( 14 years) either in LD12:12 or DD, or both, with light intensity, temperature and humidity same as that for the maintenance of populations. Fly handling and experiments in the dark were performed under dim red light $(\lambda>650 \mathrm{~nm})$.

\section{Egg-to-puparium duration assay}

Egg-to-puparium duration for all the populations was assayed under two light regimes - LD12:12 and DD. After having provided yeast paste supplemented media for three days, all populations were provided with media plates for $1 \mathrm{~h}$ as substrate for oviposition. These plates were then replaced by fresh food medium plates for the next $1 \mathrm{~h}$. Eggs laid on these plates were collected and 30 eggs were dispensed into each vial. A total of 10 such vials were used per replicate population per light regime making a total of 300 eggs per population per light regime. These vials were transferred to respective light regimes and monitored for the first pupariation event. After the first puparium was observed, vials were checked every two hours to count the number of puparia formed thereafter, and the assay was terminated when no pupariation event was seen for 24 consecutive hours. It was observed that a small proportion of larvae took relatively longer to pupariate, thus rendering the egg-to-puparium duration distribution right skewed (Fig. 1a, b; Additional file 1: Figure S3). Mean egg-topuparium duration cannot be used as a reliable measure for such distributions [62], and therefore, we used median egg-to-puparium duration (calculated as the time from egg collection for $50 \%$ of total pupariation events in a vial) for the same. The median egg-to-puparium duration was estimated for every replicate vial and then averaged across vials to obtain median egg-to-puparium duration for a given replicate population.

\section{Egg-to-adult duration assay}

Egg collection protocol and environmental conditions for the egg-to-adult duration assay were identical to eggto-puparium duration assay. After egg collection and transfer to LD12:12 or DD, eclosion of the first adult fly was monitored following which vials were subjected to two hourly checks to count the number of flies that eclosed thereafter. The assay was terminated when no eclosion event was observed for $24 \mathrm{~h}$. To facilitate comparisons between egg-to-puparium and egg-to-adult duration, we used median durations as a measure for analysis. The procedure to estimate median egg-to-adult duration was same as that described for median egg-topuparium duration.

\section{Estimation of egg-to-puparium and egg-to-adult survivorship}

Egg-collection protocol and environmental conditions for the survivorship assays were same as that for the egg-to-puparium and egg-to-adult duration assay. Proportion of 30 eggs (total number of eggs dispensed per vial for the assay) that successfully pupariated was used as a measure for egg-to-puparium survivorship, while proportion of adults that successfully eclosed was used to estimate egg-to-adult survivorship. Individuals that were stuck in the pupal case and died within the pupa were considered to not have eclosed successfully. Percentage survivorship was calculated for every replicate vial and then averaged across vials to obtain average survivorship per replicate population. 


\section{Dry-weight at pupariation}

The protocol for egg collection and subsequent environmental conditions for development under LD12:12 and DD was the same as that described for egg-to-puparium duration assay. From the initiation of the first pupariation event, freshly formed puparia (P1 stage) were collected every $2 \mathrm{~h}$ and frozen at $-20{ }^{\circ} \mathrm{C}$. These puparia were later sorted into 10 replicate groups with 5 puparia in each group; dried at $70{ }^{\circ} \mathrm{C}$ for $36 \mathrm{~h}$ after which their dry weight was assayed. Dry-weight of each group was measured at least thrice to account for instrument error and then normalized by the number of puparia $(n=5)$. The dry-weight measurements from 10 such groups were then averaged to obtain mean puparium dryweight per replicate population.

\section{Dry-weight at eclosion}

The protocol for assaying dry-weight at eclosion was the same as that for dry-weight at pupariation assay except that instead of puparia freshly eclosed (within $2 \mathrm{~h}$ of eclosion) adult flies in LD12:12 or DD were used.

\section{Fecundity assay}

The populations used in the present study are maintained on a 21 day discrete generation cycle where eggs for the next generation are collected on day 21 post egg collection (average adult age of 11 days). Since only eggs laid around this day would determine an individual's contribution to the gene pool for the next generation and consequently to its fitness, we estimated fecundity only under LD12:12 around day 11 (post-eclosion) in the progeny of standardized populations, which were collected in plexi-glass cages and maintained under LD12:12 in mixed-sex groups similar to that used for regular maintenance of populations. On day 8 (average adult age), flies from plexi-glass cages were collected, separated using mild carbon-di-oxide anesthesia and transferred into vials containing $\sim 4 \mathrm{ml} \mathrm{BJ}$ media for conditioning at a density of 10 flies/vial (5 of each sex). In parallel, additional sets of conditioning vials were set aside from which flies for pre-fecundity dry-weight assay were to be collected later (described in the following section). On day 10, flies from the conditioning vials were sorted into single male-female pairs and transferred into 20 vials/population containing $1 \mathrm{ml}$ BJ medium. After $24 \mathrm{~h}$ (day 11), flies were transferred to fresh set of vials and the same was repeated on day 12 . Average number of eggs laid per female across days 1012 was used as a measure of mean fecundity/female around day 11 . Only vials from which data could be collected for all three days were used and those in which either male or female died within the three days were not used for data analysis.

\section{Estimation of pre- and post-fecundity assay dry-weights}

To assess pre-fecundity assay dry-weight of females, 20 females (for every replicate population) from separate sets of conditioning vials (which were not used for fecundity assay) as described in the preceding section were frozen at $-20{ }^{\circ} \mathrm{C}$ at the beginning of day 10 . Additionally, at the end of the fecundity assay (end of day 12), females used for the assay were collected and frozen. All flies were then dried at $70{ }^{\circ} \mathrm{C}$ for $36 \mathrm{~h}$, sorted into groups of 5 individuals each and weighed at least thrice to estimate dry-weight/female. Dry-weight measurements were then averaged across groups to calculate mean pre- and postfecundity assay dry-weight/female/replicate population. Further, dry-weight loss during fecundity assay was estimated by calculating the difference in pre- and postfecundity assay dry-weight and was used to normalize the fecundity/female values to calculate fecundity per unit dry-weight lost as an estimate for biomass to egg conversion ratio. However, this is under the assumption that the biomass lost is entirely converted to eggs laid which may not necessarily be the case but nevertheless can be used as a proxy for assessment of for biomass-toegg conversion ratio.

\section{Longevity assay}

Longevity of flies was assayed only in LD12:12 with environmental conditions same as described previously. Freshly eclosed virgin males and females were collected from the progeny of standardized populations every $6 \mathrm{~h}$ over three consecutive days. On the fourth day, all flies of a given sex and population were mixed and randomly distributed in groups of $10 \mathrm{flies} / \mathrm{vial} / \mathrm{sex}$ into 10 replicate vials containing $\sim 4 \mathrm{ml} \mathrm{BJ}$ media. Therefore, every replicate population comprised 20 vials in total with 10 vials for each sex and each vial housing 10 flies (average age of 2 days). Thereafter, flies were transferred to fresh BJ media every 3rd day and longevity was estimated by counting the number of dead flies in each vial every $24 \mathrm{~h}$. The assay was continued until all flies died. While care was taken to ensure no flies escaped during transfers to fresh media vials, a few of them either escaped or were crushed between the cotton plug and the vial, and hence were not considered for calculating percentage survivorship for that vial. Similar to egg-to-puparium and egg-to-adult duration, longevity distribution was also right-skewed and therefore, we used median longevity (time taken for the death of $50 \%$ of individuals in a given vial) as the measure of longevity.

\section{Statistical analyses}

All measures of pre-adult duration, pre-adult survivorship, fecundity, dry-weight and longevity were estimated for each replicate vial and then averaged to obtain mean values for replicate populations. These replicate means 
served as data for statistical analyses by a randomized block design mixed model analysis of variance (ANOVA) with 'population', 'light regime,' 'stage (at which fecundity was assayed)' or 'sex' (whichever was appropriate) as fixed factors and 'replicate population' as random factor. All percentage and ratio values were arcsine square root and $\log$ transformed respectively before subjecting them to ANOVA. Post hoc multiple comparisons were performed at a significance level $(\alpha)$ of 0.05 by method of Tukey's HSD. All statistical analyses were implemented on STATISTICA for Windows, Release 5.0B (Statsoft 1995).

\section{Availability of data and materials}

The dataset(s) supporting the conclusions of this article is(are) included within the article and as supplementary online material.

\section{Additional file}

\section{Additional file 1:Figure S1. Schematic of eclosion profile of $D$.} melanogaster under laboratory LD12:12 (12 h of light and dark each) cycles at $25^{\circ} \mathrm{C}$. The shaded area represents night and the unshaded area represents day. Zeitgeber Time (ZT) depicts the time of day with ZT00 indicating lights-ON and ZT12 representing lights-OFF. Figure S2: Schematic of laboratory selection protocol employed for the early and the late populations. Zeitgeber Time (ZT) 21-00 represents the early window during which flies for the early populations are collected and ZTO913 represents the late window during which flies for the late populations are collected. Figure S3: Proportion of individuals pupariated as a function of time from egg collection for the early (panel 1), the early-control (panel 2), the control (panel 3), the late-control (panel 4) and the late (panel 5) populations in (a) LD12:12 and (b) DD. R1-R4 represents the four replicates of the respective populations used for the study. The black and white horizontal bars at the bottom represent night and day respectively. Figure S4: Proportion of individuals eclosed as a function of time from egg collection for the early (panel 1), the early-control (panel 2), the control (panel 3), the late-control (panel 4) and the late (panel 5) populations in (a) LD12:12 and (b) DD. R1-R4 represents the four replicates of the respective populations used for the study. The black and white horizontal bars at the bottom represent night and day respectively. Table S1. Median egg-to-puparium and egg-toadult duration presented as mean ( \pm SD) in hours for all populations in LD12:12 and DD light regimes. Table S2: Percentage egg-to-puparium survivorship and egg-to-adult survivorship presented as mean ( \pm SD) for all populations in LD12:12 and DD light regimes. Table S3: Average dry-weight at pupariation and at eclosion presented as mean ( \pm SD) in $\mu \mathrm{g}$ for all populations in LD12:12 and DD light regimes. Table S4: Average eggs laid/female on day 11 post-eclosion, dry-weight in $\mu \mathrm{g}$ at pre- and post-fecundity assay stages, and median longevity of all populations in LD12:12. All values are presented as mean $( \pm S D)$. (PDF $14678 \mathrm{~kb}$ )

\section{Competing interests}

The authors declare that they have no competing interests.

\section{Authors' contributions}

NKL and VKS conceived the idea and designed experiments. NKL and RK performed experiments. NKL analysed the data, and NKL and VKS wrote the manuscript. All authors read, proposed revisions and approved the manuscript.

\section{Acknowledgements}

The authors thank G Gupta, M Srivastava, P Kundu, and V Varma for their assistance with the experiments, A Lakshman for assistance with the preparation of the revised manuscript, two anonymous reviewers and Dr. Sheeba Vasu for suggesting useful changes to the manuscript. This study was funded by Scientific and Education Research Board (SERB), New Delhi,
India, and Jawaharlal Nehru Centre for Advanced Scientific Research (JNCASR), Bangalore, India.

Received: 5 November 2015 Accepted: 16 February 2016

Published online: 27 February 2016

\section{References}

1. Cloudsley-Thompson JL. Adaptive functions of circadian rhythms. Cold Spring Harb Symp Quant Biol. 1960;25:345-55.

2. Aschoff J. Adaptive cycles: their significance for defining environmental hazards. Int J Biometeorol. 1967;11:255-78.

3. Pittendrigh CS. Temporal organization: reflections of a Darwinian clock-watcher. Annu Rev Physiol. 1993;55:17-54.

4. Fleury F, Allemand R, Vavre F, Fouillet P, Boulétreau M. Adaptive significance of a circadian clock: temporal segregation of activities reduces intrinsic competitive inferiority in Drosophila parasitoids. Proc R Soc B. 2000;267:1005-10

5. Emerson K, Bradshaw WE, Holzapfel CM. Concordance of the circadian clock with the environment is necessary to maximize fitness in natural populations. Evolution. 2008;62:979-83.

6. Vaze KM, Sharma VK. On the adaptive significance of circadian clocks for their owners. Chronobiol Int. 2013;30:413-33.

7. West AC, Bechtold DA. The cost of circadian desynchrony: Evidence, insights and open questions. Bioessays. 2015;37:777-88.

8. Dunlap JC, Loros JJ, DeCoursey PJ. Chronobiology: biological timekeeping. Massachusetts, U S A: Sinauer Associates; 2004.

9. Saunders DS. Insect clocks. Amsterdam: Elsevier; 2002.

10. DeCoursey PJ, Krulas JR, Mele G, Holley DC. Circadian performance of suprachiasmatic nuclei (SCN)-lesioned antelope ground squirrels in a desert enclosure. Physiol Behav. 1997;62:1099-108.

11. DeCoursey PJ, Krulas JR. Behavior of SCN-lesioned chipmunks in natural habitat: a pilot study. J Biol Rhythms. 1998;13:229-44.

12. DeCoursey PJ, Walker JK, Smith SA. A circadian pacemaker in free-living chipmunks: essential for survival? J Comp Physiol A. 2000;186:169-80.

13. Daan S, Spoelstra K, Albrecht U, Schmutz I, Daan M, Daan M, et al. Lab mice in the field: unorthodox daily activity and effects of a dysfunctional circadian clock allele. J Biol Rhythms. 2011;26:118-29.

14. Allemand RY, Cohet Y, David J. Increase in the longevity of adult Drosophila melanogaster kept in permanent darkness. Exp Gerontol. 1973;8:279-83.

15. Kumar S, Mohan A, Sharma VK. Circadian dysfunction reduces lifespan in Drosophila melanogaster. Chronobiol Int. 2005;22:641-53.

16. Beaver LM, Gvakharia BO, Vollintine TS, Hege DM, Stanewsky R, Giebultowicz JM. Loss of circadian clock function decreases reproductive fitness in males of Drosophila melanogaster. Proc Natl Acad Sci U S A. 2002:99:2134-9.

17. Beaver LM, Rush BL, Gvakharia BO, Giebultowicz JM. Non-circadian regulation and function of clock genes period and timeless in oogenesis of Drosophila melanogaster. J Biol Rhythms. 2003;18:463-72.

18. Blume J, Bünning E, Gunzler E. Zur aktivitätsperiodik bei höhlentieren. Naturwissenschaften. 1962:49:525

19. Poulson TL, White WB. The cave environment. Science. 1969:165:971-81.

20. Lankinen P. Geographical variation in circadian eclosion rhythm and photoperiodic adult diapause in Drosophila littoralis. J Comp Physiol A. 1986;159:123-42

21. Lankinen P. North-south differences in circadian eclosion rhythm in European populations of Drosophila subobscura. Heredity. 1993;71:210-8.

22. Pittendrigh CS, Takamura T. Latitudinal clines in the properties of a circadian pacemaker. J Biol Rhythms. 1989;4:217-35.

23. Costa R, Peixoto AA, Barbujani G, Kyriacou CP. A latitudinal cline in a Drosophila clock gene. Proc R Soc B. 1992;250:43-9.

24. Rosato E, Peixoto AA, Barbujani G, Costa R, Kyriacou CP. Molecular polymorphism in the period gene of Drosophila simulans. Genetics. 1994;138:693-707.

25. Sawyer LA, Hennessy JM, Peixoto AA, Rosato E, Parkinson H, Costa R, et al. Natural variation in a Drosophila clock gene and temperature compensation. Science. 1997;278:2117-20.

26. Sawyer LA, Sandrelli F, Pasetto C, Peixoto AA, Rosato E, Costa $R$, et al. The period gene Thr-Gly polymorphism in Australian and African Drosophila melanogaster populations: implications for selection. Genetics. 2006;174:465-80.

27. Michael TP, Salomé PA, Yu HJ, Spencer TR, Sharp EL, McPeek MA, et al. Enhanced fitness conferred by naturally occurring variation in the circadian clock. Science. 2003;302:1049-53. 
28. Tauber E, Zordan M, Sandrelli F, Pegoraro M, Osterwalder N, Breda C, et al. Natural selection favors a newly derived timeless allele in Drosophila melanogaster. Science. 2007;316:1895-8.

29. Skopik SD, Pittendrigh CS. Circadian systems II. The oscillation in the individual Drosophila pupa; its independence of developmental stage. Proc Natl Acad Sci U S A. 1967:58:1862-9.

30. De J, Varma V, Sharma VK. Adult emergence rhythm of fruit flies Drosophila melanogaster under seminatural conditions. J Biol Rhythms. 2012;27:280-6.

31. Pittendrigh CS. Circadian systems I. The driving oscillation and its assay in Drosophila pseudoobscura. Proc Natl Acad Sci U S A. 1967;58:1762-7.

32. Pittendrigh CS, Minis DH. The photoperiodic time measurement in Pectinophora gossypiella and its relation to the circadian system in that species. In: Menaker M, editor. Biochronometry. Washington DC: Natl Acad Sci; 1971. p. 212-50.

33. Duffy JF, Dijk DJ, Hall EF, Czeisler CA. Relationship of endogenous circadian melatonin and temperature rhythms to self-reported preference for morning or evening activity in young and older people. J Investig Med. 1999;47:141-50.

34. Duffy JF, Rimmer DW, Czeisler CA. Association of intrinsic circadian period with morningness-eveningness, usual wake time, and circadian phase. Behav Neurosci. 2001;115:895-9.

35. Roenneberg T, Wirz-Justice A, Merrow M. Life between clocks: daily temporal patterns of human chronotypes. J Biol Rhythms. 2003;18:80-90.

36. Kumar S, Kumar D, Paranjpe DA, Akarsh RC, Sharma VK. Selection on the timing of adult emergence results in altered circadian clocks in fruit flies Drosophila melanogaster. J Exp Biol. 2007;210:906-18.

37. Nikhil KL, Vaze KM, Ratna K, Sharma VK. Circadian clock properties of fruit flies Drosophila melanogaster exhibiting early and late emergence chronotypes. Chronobiol Int. 2016; In press.

38. Vaze KM, Nikhil KL, Abhilash L, Sharma VK. Early- and late-emerging Drosophila melanogaster fruit flies differ in their sensitivity to light during morning and evening. Chronobiol Int. 2012;29:674-82.

39. Nikhil KL, Goirik G, Ratna K, Sharma VK. Role of temperature in mediating morning and evening emergence chronotypes in fruit flies Drosophila melanogaster. J Biol Rhythms. 2014;29:427-41.

40. Miyatake T. Correlated responses to selection for developmental period in Bactrocera cucurbitae (Diptera: Tephritidae): time of mating and daily activity rhythms. Behav Genet. 1997;27:489-98.

41. Shimizu T, Miyatake T, Watari Y, Arai T. A gene pleiotropically controlling developmental and circadian periods in the melon fly, Bactrocera cucurbitae (Diptera: Tephritidae). Heredity. 1997;79:600-5.

42. Kyriacou CP, Oldroyd M, Wood J, Sharp M, Hill M. Clock mutations alter developmental timing in Drosophila. Heredity. 1990;64:395-401.

43. Takahashi KH, Teramura K, Muraoka S, Okada Y, Miyatake T. Genetic correlation between the pre-adult developmental period and locomotor activity rhythm in Drosophila melanogaster. Heredity. 2013;110:312-20.

44. Yadav P, Sharma VK. Circadian clocks of faster developing fruit fly populations also age faster. Biogerontology. 2013;15:33-45.

45. Kumar S, Vaze KM, Kumar D, Sharma VK. Selection for early and late adult emergence alters the rate of pre-adult development in Drosophila melanogaster. BMC Dev Biol. 2006;6:57-71.

46. Paranjpe DA, Anitha D, Chandrashekaran MK, Joshi A, Sharma VK. Possible role of eclosion rhythm in mediating the effects of light-dark environments on preadult development in Drosophila melanogaster. BMC Dev Biol. 2005;5:5-10.

47. Yadav P, Thandapani M, Sharma VK. Interaction of light-regimes and circadian clocks modulate timing of pre-adult developmental events in Drosophila. BMC Dev Biol. 2014;14:19-31.

48. Qiu J, Hardin PE. Developmental state and the circadian clock interact to influence the timing of eclosion in Drosophila melanogaster. J Biol Rhythms. 1996;11:75-86

49. Mukherjee N, Kannan NN, Yadav P, Sharma VK. A model based on the oscillatory threshold and build-up of a developmental substance can explain gating of adult emergence in fruit flies Drosophila melanogaster. J Exp Biol. 2012; 215:2960-8

50. Pittendrigh CS, Skopik SK. Circadian systems V. The driving oscillation and the temporal sequence of development. Proc Natl Acad Sci U S A. 1970;65:500-7.

51. Konopka RJ, Benzer S. Clock mutants of Drosophila melanogaster. Proc Natl Acad Sci U S A. 1971:68:2112-6.

52. Robertson FW. The ecological genetics of growth in Drosophila. 1. Body size and development time on different diets. Genet Res Camb. 1960;1:288-304.

53. Robertson FW. The ecological genetics of growth in Drosophila. 6. The genetic correlation between the duration of the larval period and body size in relation to larval diet. Genet Res Camb. 1963;4:74-92.
54. Hillesheim E, Stearns SC. Correlated responses in life-history traits to artificial selection for body weight in Drosophila melanogaster. Evolution. 1992;46:745-52.

55. Partridge L, Fowler K. Direct and correlated responses to selection on age at reproduction in Drosophila melanogaster. Evolution. 1992;46:76-91.

56. Rose MR. Laboratory evolution of postponed senescence in Drosophila melanogaster. Evolution. 1984;38:1004-10.

57. Chippindale AK, Leroi A, Kim SB, Rose MR. Phenotypic plasticity and selection in Drosophila life history evolution. 1. Nutrition and the cost of reproduction. J Evol Biol. 1993;6:171-93.

58. Partridge $\mathrm{L}$, Barton $\mathrm{NH}$. Evolution of aging: Testing the theory using Drosophila. Genetica. 1993;91:89-98.

59. Yadav P, Sharma VK. Correlated changes in circadian clocks in response to selection for faster pre-adult development in fruit flies Drosophila melanogaster. J Comp Physiol B. 2013;183:333-43.

60. Yadav $P$, Sharma VK. Correlated changes in life history traits in response to selection for faster pre-adult development in the fruit fly Drosophila melanogaster. J Exp Biol. 2014;217:580-9.

61. Garland Jr T, Adolph SC. Physiological differentiation of vertebrate populations. Annu Rev Ecol Evol Syst. 1991;22:193-228.

62. Sokal RR, Rohlf FJ. Biometry: The principles and practices of statistics in biological research. New York: WH Freeman; 1995.

\section{Submit your next manuscript to BioMed Central and we will help you at every step:}

- We accept pre-submission inquiries

- Our selector tool helps you to find the most relevant journal

- We provide round the clock customer support

- Convenient online submission

- Thorough peer review

- Inclusion in PubMed and all major indexing services

- Maximum visibility for your research

Submit your manuscript at www.biomedcentral.com/submit
) Biomed Central 